\title{
Cell type and context-specific function of PLAG1 for IGF2 P3 promoter activity
}

\author{
MONIRA AKHTAR ${ }^{1}$, CLAES HOLMGREN ${ }^{1}$, ANITA GÖNDÖR ${ }^{3}$, MATTIAS VESTERLUND ${ }^{2}$, \\ CHANDRASEKHAR KANDURI $^{4,5}$, CATHARINA LARSSON ${ }^{2}$ and TOMAS J. EKSTRÖM ${ }^{1}$ \\ ${ }^{1}$ Department of Clinical Neuroscience, Karolinska Institutet, Karolinska University Hospital; \\ ${ }^{2}$ Department of Molecular Medicine and Surgery, Karolinska Institutet, CMM L8:01, SE-171 76 Stockholm; \\ ${ }^{3}$ Microbiology and Tumor Center; Karolinska Institutet, SE-171 77 Stockholm; ${ }^{4}$ Department of Genetics \\ and Pathology, Uppsala University, Rudbeck Laboratory, SE-751 85 Uppsala, Sweden
}

Received June 15, 2012; Accepted August 14, 2012

DOI: 10.3892/ijo.2012.1641

\begin{abstract}
The fetal transcription factor PLAG1 is found to be overexpressed in cancers, and has been suggested to bind the insulin like growth factor 2 (IGF2) P3 promoter, and to activate the $I G F 2$ gene. The expression of $I G F 2$ has partly been linked to loss of CTCF-dependent chromatin insulator function at the $H 19$ imprinting control region (ICR). We investigated the role of PLAG1 for IGF2 regulation in Hep3B and JEG-3 cell lines. Chromatin immunoprecipitation revealed cell type-specific binding of PLAG1 to the IGF2 P3 promoter, which was substantially insensitive to recombinant PLAG1 overexpression in the endogenous context. We hypothesized that the $H 19$ chromatin insulator may be involved in the cell type-specific PLAG1 response. By using a GFP reporter gene/ insulator assay plasmid construct with and without the $\mathrm{H} 19$ ICR and/or an SV40 enhancer, we confirm that the effect of the insulator is specifically associated with the activity of the $I G F 2$ P3 promoter in the GFP reporter system, and furthermore, that the reporter insulator is functional in JEG-3 but not in Hep3B cells. FACS analysis was used to assess the function of PLAG1 in low endogenously expressing, but Zn-inducible stable PLAG1 expressing JEG-3 cell clones. Considerable increase in IGF2 expression upon PLAG1 induction with a partial insulator overriding activity was found using the reporter constructs. This is in contrast to the effect of the endogenous IGF2 gene which was insensitive to PLAG1 expression in JEG-3, while modestly
\end{abstract}

Correspondence to: Dr Tomas Ekström, Department of Clinical Neuroscience, Karolinska Institutet, Karolinska University Hospital, CMM L8:01, SE-171 76 Stockholm, Sweden

E-mail: tomas.ekstrom@ki.se

Present address: ${ }^{5}$ Department of Medical and Clinical Genetics, Institute of Biomedicine, Sahlgrenska Academy, University of Gothenburg, SE-413 90 Gothenburg, Sweden

Key words: H19 insulator, PLAG1, IGF2, P3 promoter, chromatin immunoprecipitation, Hep3B, JEG-3 induced the already highly expressed $I G F 2$ gene in Hep3B cells. We suggest that the PLAG1 binding to the IGF2 $\mathrm{P} 3$ promoter and $I G F 2$ expression is cell type-specific, and that the PLAG1 transcription factor acts as a transcriptional facilitator that partially overrides the insulation by the H19 ICR.

\section{Introduction}

The insulin like growth factor (IGF) system is composed of two related growth factors (IGF-I and IGF-II) and a group of IGF binding proteins (IGFBPs). This system has important roles in human development and maintenance of normal functions of many cells of the mammalian organism. In human, both IGF-I and IGF-II are produced in multiple tissues throughout life and have potentially divergent roles in pathophysiology. IGF-II has various roles in pathological conditions and a particularly prominent role in tumor development. This association between cancer and IGFs has long been a subject for investigation (1). Dysregulation of $I G F 2$, its receptors (the type 1 and 2 IGFR), and IGFBPs provide part of the underlying mechanism for uncontrolled increase in cellular proliferation, as is evident in cancer. The IGF2 locus shows loss of imprinting (LOI) in several types of cancer (reviewed in ref. 2) and it was shown to be a marker for colorectal carcinoma risk (3).

Genome wide epigenetic marks are maintained with high fidelity in normal cells but frequently become destabilized in human cancer $(4,5)$. This is exemplified by the observations that the normally silenced state of the maternally inherited IGF2 allele is lost in several cancer forms, such as Wilms' tumor (6) and colon cancer (7), resulting in LOI and an activation of biallelic expression patterns. The finding that $I G F 2 \mathrm{LOI}$ in peripheral blood cells is the earliest predictive marker for colon cancer reinforces the notion that constitutive epigenetic lesions predispose for cancer (3). The normally repressed states of the paternal $H 19$ and the maternal IGF2 alleles are coordinated by the differentially methylated imprinting control region (ICR or DMR) in the 5'-flank of the $H 19$ gene (8). This feature involves a methylation-sensitive, long-range chromatin insulator (9), that via target sites for the 11 zinc finger protein CTCF represses the maternal IGF2 allele in mammals $(10,11)$. 
It has further been shown that in mouse, this insulator region is in physical contact with the promoter region of the Igf 2 gene $(10,12)$ and that cohesin is maintaining the insulation properties in the H19/IGF2 locus (13). In essence, the binding of CTCF to the ICR, prevents the Igf $2 / I G F 2$ gene from being in close proximity to the enhancers located in the 3'-flank of the $H 19$ gene, while they can interact with the $H 19$ promoter. The absence of CTCF binding lets the same enhancers interact with the Igf2/IGF2 gene. The fact that the binding of CTCF to the ICR is methylation sensitive further strengthens the notion that the methylation status of the ICR is the most crucial key for proper maintenance of the mono-allelic expression patterns of these genes.

Reports have shown that the CTCF target sites within the H19 ICR are de novo methylated in a wide range of human cancers $(14,15)$, which would lead to prevention of CTCF binding and loss of insulator function at the maternal allele, followed by IGF2 allelic reactivation. This phenomenon can, however, not explain the appearance of LOI of IGF2 in correlation with hypomethylation at the maternal ICR which has been reported in colon cancer (16) and bladder cancer (17). These findings suggest alternative, ICR independent, mechanisms that can reactivate the silenced $I G F 2$ gene on the maternal allele.

PLAG1 is a proto-oncogene discovered in pleomorphic adenoma of the salivary glands (18). It has been described as a seven zinc finger transcription factor which is developmentally regulated and expressed during fetal development in several tissues, but is downregulated after birth. One reported mechanism for deregulation of PLAGl is promoter swapping with the $\beta$-catenin gene, resulting in ectopic expression of PLAG1 in pleomorphic adenoma of the salivary glands (18). PLAG1 has been shown to have oncogenic capacity and to potently trigger IGF 2 expression by its binding to the $\mathrm{P} 3$ promoter as shown by electromobility shift assay (EMSA) (19). In addition PLAGI has been shown to be overexpressed in several other tumor types, such as hepatoblastoma (20), lipoblastoma (21) and acute myeloid leukemia (22).

Here, we set out to investigate the role of PLAG1 in the regulation of $I G F 2$ in an insulator-reporter system as well as at the endogenous level in cell lines. The findings provide evidence that PLAG1 can activate IGF2 P3 promoterdependent expression, despite the presence of the ICR in a reporter system. The endogenous sensitivity of $I G F 2$ to PLAG1 displayed a cell type-specific response, and the IGF2 P3 promoter revealed clear differential capacity to bind the PLAG1 transcription factor which was however, not reflected by the $I G F 2$ transcription.

\section{Materials and methods}

Cell lines and stably transfected cell clones. JEG-3 (human choriocarcinoma) and Hep3B (human hepatocellular carcinoma) cell lines were maintained as previously described (23). For stable transfection, $2 \mu \mathrm{g}$ of the pSAR-PLAG1 (19) construct and $0.1 \mu \mathrm{g}$ of the pBK-CMV plasmid (neo + ) was co-transfected into JEG-3 cells using Fugene 6 (Roche) according to the protocol recommended by the manufacturer, followed by neomycin selection $(500 \mu \mathrm{M})$ for 4 weeks. Resistant cell clones were picked, expanded and analysed for zinc-inducible $(100 \mu \mathrm{M}$ $\left.\mathrm{ZnCl}_{2}\right) P L A G 1$ expression using quantitative real-time PCR
(qRT-PCR). One zinc responsive cell clone which had undetectable PLAG1 mRNA in the absence of zinc was selected for further experimental procedures.

Quantitative real-time PCR analysis of IGF2 and PLAG1. qRT-PCR was performed to evaluate the IGF2 and PLAGI mRNA levels in wild-type Hep3B and JEG-3 cells. Total-RNA was extracted using Trizol and phenol-chloroform, quantified by Nanodrop, and 6-9 $\mu \mathrm{g}$ of RNA was treated with DNase I (6-7 units, at $37^{\circ} \mathrm{C}$ for $\left.30 \mathrm{~min}\right)$. DNase I treated RNA $(1 \mu \mathrm{g})$ was used for cDNA synthesis with iScript cDNA synthesis kit (Bio-Rad) in $20 \mu \mathrm{l}$ reactions. Reverse transcription was performed in $20 \mu \mathrm{l}$ reactions following the protocol from the manufacturer. A total of $2 \mu \mathrm{l}$ of product was used for PCR amplification of individual transcripts from IGF2 and PLAG1 in an Applied Biosystem 7500 Fast Real-Time PCR instrument using the iScrip SYBR-Green kit (Bio-Rad). The primers for IGF2 are F: 5'-AAC TGG CCA TCC GAA AAT AGC and R: 5'-TTT GCA TGG ATT TTG GTT TTC AT. For PLAG1 primers sequences are F: 5'-CAC ACA GGA GAG AGG CCC TAC and R: 5'-CAC AAT AAT TAC ACT TGT. Thermo cycling conditions were $50^{\circ} \mathrm{C}$ for $2 \mathrm{~min}$ and $95^{\circ} \mathrm{C}$ for $5 \mathrm{~min}$, followed by 40 cycles of $95^{\circ} \mathrm{C}$ for $20 \mathrm{sec}$ and $57^{\circ}$ or $67^{\circ} \mathrm{C}$ for $30 \mathrm{sec}$ for $I G F 2$ and $P L A G 1$, respectively. The GAPDH mRNA was used for normalization to correct for the amount of RNA loaded in each sample. Primer sequences are F: 5'-GAA GGT GAA GGT CGG AGT C and R: 5'-GAA GAT GGT GAT GGG ATT TC. Thermo cycling conditions were $50^{\circ} \mathrm{C}$ for $2 \mathrm{~min}$ and $95^{\circ} \mathrm{C}$ for $5 \mathrm{~min}$, followed by 40 cycles of $95^{\circ} \mathrm{C}$ for $20 \mathrm{sec}$ and $60^{\circ} \mathrm{C}$ for $30 \mathrm{sec}$.

Semi-quantitative RT-PCR. Semi-quantitative RT-PCR of PLAG1 transcripts was performed in JEG-3 stable clones before and after $\mathrm{Zn}$-induction using the primers mentioned above. Thermo cycling conditions were $94^{\circ} \mathrm{C}$ for $5 \mathrm{~min}$, followed by 30 cycles of $94^{\circ} \mathrm{C}$ for $30 \mathrm{sec}, 59^{\circ} \mathrm{C}$ for $50 \mathrm{sec}$ and $70^{\circ} \mathrm{C}$ for $50 \mathrm{sec}$ and final extension at $72^{\circ} \mathrm{C}$ for $10 \mathrm{~min}$.

Transient transfection and expression of IGF2 P3 driven GFP reporter constructs. The IGF2 $\mathrm{P} 3$ promoter was cloned as a BamHI-SalI fragment into the BglII-SalI site of the pd2EGFP-1 vector (Clontech) to create the pd2EGFP1-IGF2-P3-GFP intermediate vector. The IGF2-P3-GFP cassette was excised from the intermediate vector by digesting with Eco47III-SspI and cloned into a Bstz17I digested pREP4 H19A vector (23) to create the $\mathrm{pA}-\mathrm{GFP}$ plasmid. The $\mathrm{pB}-\mathrm{GFP}$ was obtained by cloning a $3.3 \mathrm{~kb} K p n \mathrm{I}-\mathrm{XhoI}$ mouse H19 ICR fragment into pA-GFP. The pO-GFP plasmid was generated by deleting the SV40 enhancer from the pA-GFP plasmid by ClaI digestion followed by re-ligation. pRep9RFP was created by cloning a KpnI-XhoI fragment containing the RFP-gene (derived from pDsRed1, Clontech) into the pRep9 vector (Invitrogen).

Equimolar amounts $(3 \mu \mathrm{g})$ of the GFP constructs were co-transfected with $0.25 \mu \mathrm{g}$ pDsRed2-C1 (Clonetech) or $1 \mu \mathrm{g}$ pRep9RFP into JEG-3 and Hep3B cells using Fugene 6 (Roche) according to the protocol recommended by the manufacturer. GFP expression was analysed using confocal microscopy (Leica DM Irbe) and flow cytometry (BD FACSCalibur), whereby the expression levels of GFP were estimated as a ratio between GFP and RFP expression (constant). 
Chromatin conformation analysis. Nuclei from Hep3B and JEG-3 cells, transfected with the pB-GFP construct, were isolated and DNase I treated as previously described (23). DNase I treated DNA $(20 \mu \mathrm{g})$ was digested with StuI and analysed in $1.7 \%$ agarose gels followed by Southern blotting according to standard procedures. The chromatin conformations were analysed by indirect end-labelling as has been accounted for before (23). The chromatin conformation was analysed by using a $170 \mathrm{bp}$ StuI-EcoRI fragment spanning the upstream region outside of the ICR as previously described (9).

Transient transfection of PLAG1. Hep3B and JEG-3 cells were transiently transfected with $3 \mu \mathrm{g}$ of the PLAG1 expression vector pCAGGS-PLAG1 (20) on 60-mm plates for $72 \mathrm{~h}$. As negative control, mock transfection with a $\beta$-galactosidase containing plasmid was done in parallel. Transfection was performed using the Lipofectamine and Plus reagent transfection kit (Invitrogen) according to the manufacturer's protocol.

Chromatin immunoprecipitation (ChIP) analysis. Binding of PLAG1 to the IGF2 P3 promoter was investigated in Hep3B and JEG-3 cells using Chromatin Immunoprecipitation (ChIP). ChIP was performed from total cellular extracts according to the Quick and Quantitative Chromatin Immunoprecipitation (Q2 ChIP) method (described in ref. 24). After trypsinization cells were cross-linked with $1 \%$ formaldehyde and chromatin was sheared by Bioruptor 10 times (10 sec each) to fragments of 200 to 1,000 base pairs and cleared for cellular debris by sedimentation. The supernatant was used for immunoprecipitation of specific protein-DNA complex using a PLAG1 antibody (Sigma, cat no. AV 38177) and normal rabbit IgG coupled to Dyna beads protein-G (Invitrogen) at $4^{\circ} \mathrm{C}$ overnight. $\mathrm{IgG}$ was used as a control for non-specific binding due to $\mathrm{Fc}$ receptor binding or other protein-protein interactions. ChIP complexes were washed with RIPA buffer and subsequently cross-links were reversed, proteins digested and DNA eluted in elution buffer. DNA was purified with PCR Purification Kit (Qiagen). The immunoprecipitated DNA was then quantified by SYBR-Green qPCR using primers flanking the PLAG1 consensus binding site in the IGF2 P3 promoter region (20). Primer sequences are F: 5'-CTGCCTGCCCGGAGAC and R: 5'-CATGCTGAATGCCCGTTCT. Thermo cycling conditions were $50^{\circ} \mathrm{C}$ for $2 \mathrm{~min}$ and $95^{\circ} \mathrm{C}$ for $5 \mathrm{~min}$, followed by 40 cycles of $95^{\circ} \mathrm{C}$ for $20 \mathrm{sec}$ and $65^{\circ} \mathrm{C}$ for $30 \mathrm{sec}$.

\section{Results}

Expression of IGF2 and PLAG1 in cell lines. Since Hep3B and JEG-3 cells were used as model systems, we assessed the endogenous mRNA levels of PLAGI and IGF2 by qRT-PCR using the housekeeping gene GAPDH as normalization factor. Substantially higher level of IGF2 (around 6-fold) was found in Hep3B as compared to JEG-3 cells (Fig. 1A). PLAG1 expression level was approximately 3 times higher in Hep3B compared to JEG-3 cells (Fig. 1B). We therefore investigated whether PLAGl expression may be an important player for higher expression of $I G F 2$ in Hep3B cells, and if transient overexpression of PLAG1 could elevate $I G F 2$ transcription. For this purpose we transfected Hep3B and JEG-3 cells with a PLAG1 expression plasmid. In Hep3B cells, a monumental 2,000-fold
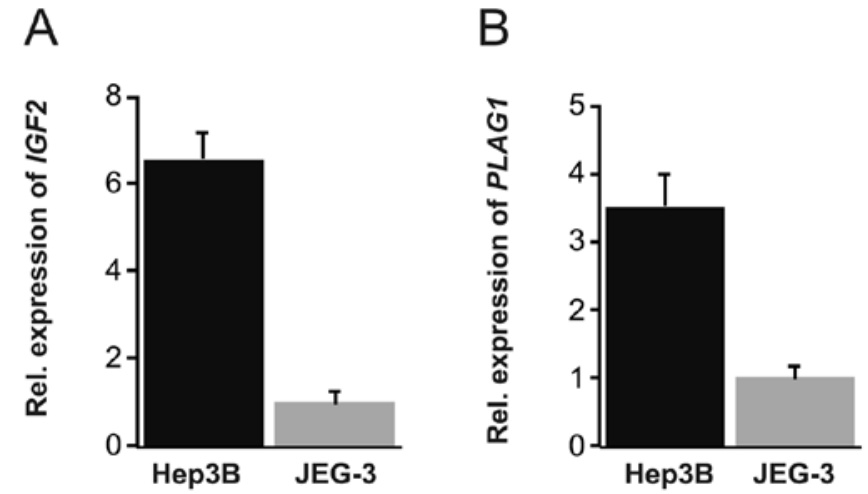

Figure 1. Detection of $I G F 2$ and PLAG1 transcripts in Hep3B and JEG-3 cells. (A) qRT-PCR analysis of the relative expression level of $I G F 2$ mRNA. (B) Real-time qPCR analysis of PLAG1 mRNA. All experiments were run in triplicate. Error bars denote standard error of the mean.

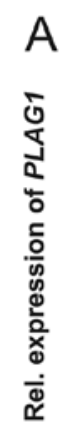
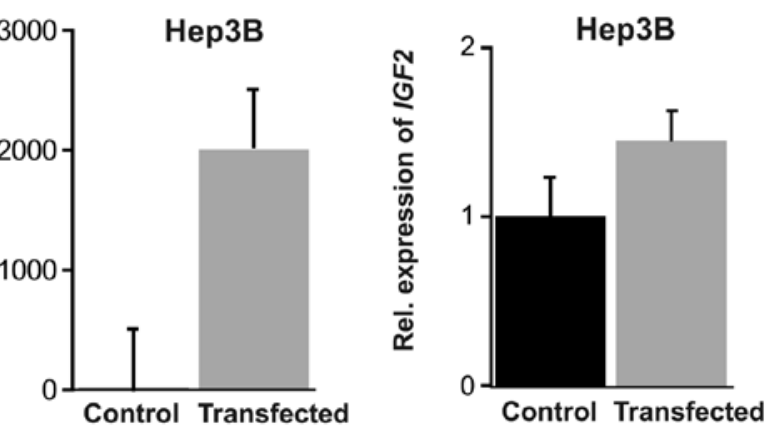

Figure 2. Detection of $I G F 2$ and PLAG1 expression in Hep3B cells following transfection with the PLAG1 expression vector, pCAGGS-PLAG1. Cells were harvested $72 \mathrm{~h}$ post-transfection. (A) PLAG1 transcripts relative to mock transfected control. (B) $I G F 2$ transcript after $P L A G 1$ overexpression, relative to mock transfected control. The experiments were run in triplicate. Error bars denote standard error of the mean.

induction of PLAG1 mRNA was achieved $(\mathrm{P}=0.0026)$, together with a modest increase of IGF2 expression (Fig. 2). In JEG-3 cells, the transfection was less efficient in comparison to Hep3B, but a robust 130-fold increase in PLAG1 mRNA was achieved $(\mathrm{P}=0.017)$. This did not, however, result in any enhanced $I G F 2$ expression (Fig. 3).

ChIP analysis. The finding that ectopic PLAG1 expression slightly induced endogenous $I G F 2$ expression in already high $I G F 2$ expressing Hep3B cells, but not in JEG-3 cells, prompted us to analyze binding of PLAG1 to the IGF2 P3 promoter using chromatin immunoprecipitation. Mock transfected Hep3B cells did not display increased binding of PLAG1 to the P3 promoter. However, transient transfection with PLAG1 resulted in increased binding (Fig. 4). The situation for JEG-3 cells was dramatically different. No binding of PLAG1 to the P3 promoter could be detected in non-transfected, or PLAG1 transfected cells (data not shown), consistent with the low endogenous $I G F 2$ expression and the inability to boost this by additional expression of PLAG1 (Fig. 3). 
A

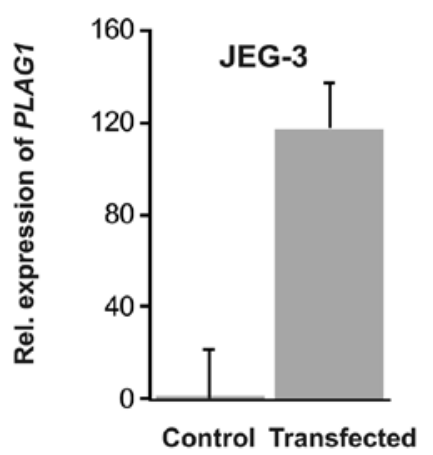

B

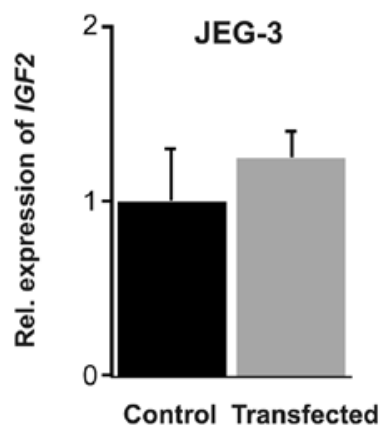

Figure 3. Detection of IGF2 and PLAG1 expression in JEG-3 cells following transfection with the PLAG1 expression vector, pCAGGS-PLAG1. Cells were harvested 72 h post-transfection. (A) PLAG1 transcripts relative to mock transfected control. (B) IGF2 transcripts after PLAG1 overexpression, relative to mock transfected control. The experiments were run in triplicate. Error bars denote standard error of the mean.

IGF2 P3 promoter reporter expression in the presence of a functional insulator. Our results on the relatively weak and cell type-specific endogenous relationship between $I G F 2$ expression and PLAG1 binding to the $\mathrm{P} 3$ promoter, prompted us to turn to a simpler system that allowed us to separate the $\mathrm{P} 3$ promoter from the important H19 3'-enhancers. We hypothesized that Hep3B and JEG-3 cells have different sensitivity to the $H 19$ upstream insulator in the regulation of $I G F 2 \mathrm{P} 3$ promoter and that PLAGI might be involved in this.

In order to investigate the insulator effect of the ICR, we developed a GFP based assay system (Fig. 5A) where we could analyze the effects of insulator function in real-time using confocal microscopy as well as expression analysis using FACS. JEG-3 and Hep3B cells were simultaneously transfected with the P3/GFP episomal constructs (Fig. 5A) and analyzed at 48h post-transfection. In JEG-3 cells the GFP expression was low in the enhancer-free promoter construct, pO-GFP, and substantially higher in the SV40 enhancer containing construct, pA-GFP (Fig. 5A). The construct which also contains an insulator, pB-GFP, showed an almost total enhancer insulation of GFP expression, as expected (Fig. 5A). The Hep 3B cell line, however, displayed a different expression pattern. As in JEG-3 cells, GFP expression was low in the enhancer-less promoter construct pO-GFP and robust in the enhancer-containing pA-GFP construct. However, in contrast to JEG-3 cells, this high expression was not attenuated in Hep3B for the insulator containing construct pB-GFP (Fig. 5A). The possible explanation that the insulator was non-functional by the absence of binding by CTCF at the ICR, was ruled out, since the chromatin conformations at the CTCF target-sites of the transfected H19 ICR were virtually identical, in JEG-3 cells and Hep3B cells (Fig. 5B).

To analyse if this aberrant expression of GFP was promoter-dependent, the IGF2 $\mathrm{P} 3$ promoter was replaced with the $H 19$ promoter in the otherwise identical constructs. FACS analyses verified that the insulator function was restored in Hep3B with these constructs, suggesting that the loss of insulator function is IGF2 $\mathrm{P} 3$ promoter-dependent (data not shown). This finding is further supported by previous observations where the ICR has been shown to be a potent insulator of the $H 19$ promoter

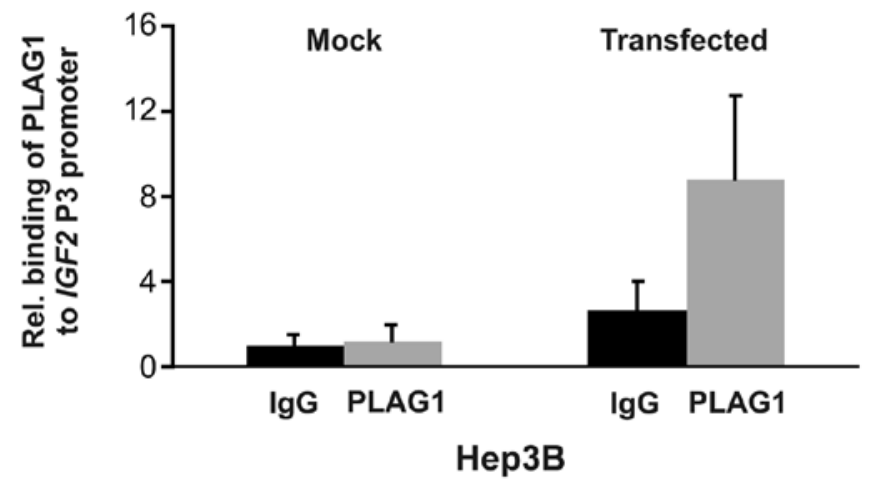

Figure 4. Analyses of PLAG1 binding at the IGF2 P3 promoter in Hep3B cells as determined by ChIP. Chromatin fragments were immunoprecipitated with anti-PLAG1 antibody and IgG as a control, and quantified by SYBR-Green qPCR. The ChIP analyses were run in triplicate and error bars denote standard error of the mean.

in Hep3B (23). Since it was previously shown that PLAG1 can upregulate $I G F 2$ expression and that binding to the $\mathrm{P} 3$ promoter could occur in an EMSA (19), together with our expression and ChIP results above, we hypothesized that PLAG1 might be involved in the cell type-specific expression of $I G F 2$ that involves the H19 ICR.

PLAG1 expression attenuates the insulator function of the $\mathrm{H} 19$ $I C R$. Since the expression of PLAGl was significantly lower in JEG-3 than in Hep3B (Fig. 1B), and the GFP-reporter construct displayed insulator activity in JEG-3 but not Hep3B cells, we examined whether increased PLAG1 expression in JEG-3 would result in a Hep3B-like situation with the GFP reporter construct. We created a zinc-inducible PLAGl cell clone derived from JEG-3 by the stable incorporation of the PSAR-PLAGI construct (19). Several cell clones were analysed and one zincinducible clone, but with undetectable PLAGI expression in the absence of $\mathrm{ZnCl}_{2}$, was selected for the subsequent analyses. Upon induction with $\mathrm{ZnCl}_{2}$ the level of PLAG1 expression increased substantially in this clone (Fig. 6A). The episomal GFP constructs (Fig. 5A) were co-transfected with RFP control constructs into the PLAGI inducible JEG-3 cell line, followed by qRT-PCR analysis of GFP expression. Upon Zn-induction of PLAGl, the expression of the GFP reporter gene was considerably elevated in all constructs (Fig. 6B). By employing the enhancer- and insulator-free construct (pO-GFP) it was shown that after $48 \mathrm{~h}$ of induction, PLAG1 can elevate the levels of transcription from the $\mathrm{P} 3$ promoter to levels similar to the enhancer driven construct (pA-GFP) in low-PLAG1 expressing cells. In the presence of the enhancer, PLAG1-induction dramatically increased the P3 driven GFP expression. Interestingly, PLAG1 could also induce high expression in the insulator-containing pB-GFP construct. Although the insulator still appears to maintain an ability to attenuate enhancer-promoter interaction in the presence of PLAGI overexpression (Fig. 6B, pB-GFP construct, $\mathrm{Zn} 48 \mathrm{~h}$ ), the level of this expression is higher than both the induced pO-GFP construct (Fig. 6B, +Zn 48 h), and more importantly, even from the non-induced enhancer-containing construct lacking an insulator, pA-GFP (Fig. 6B, -Zn). Thus, after $48 \mathrm{~h}$ of 

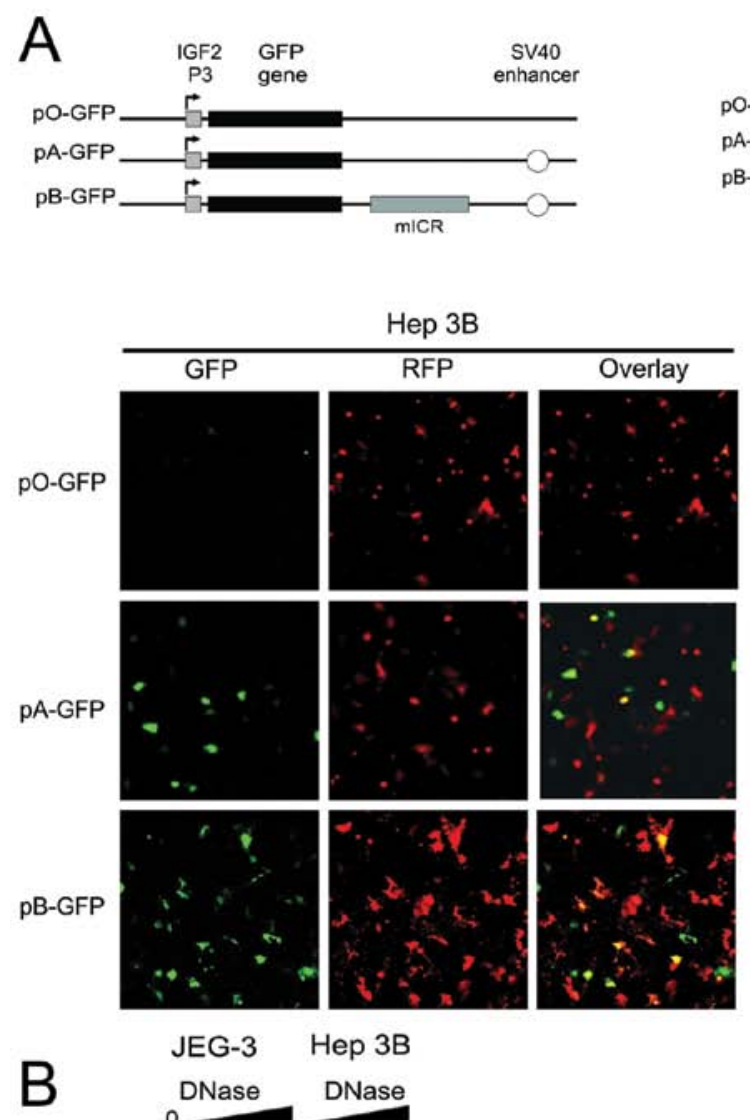
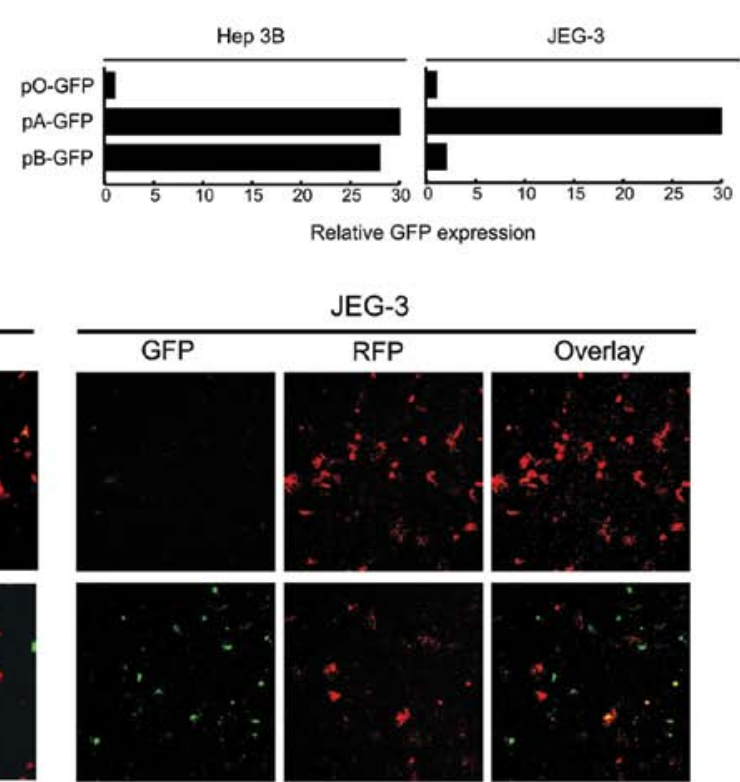

JEG-3
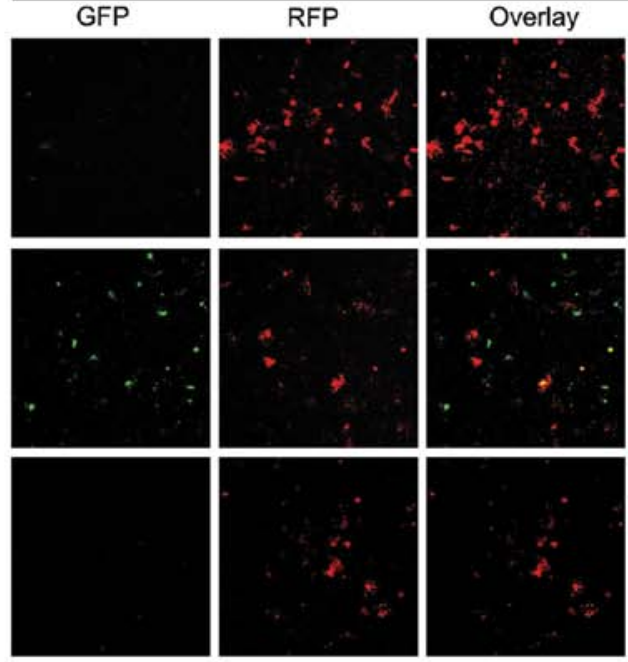

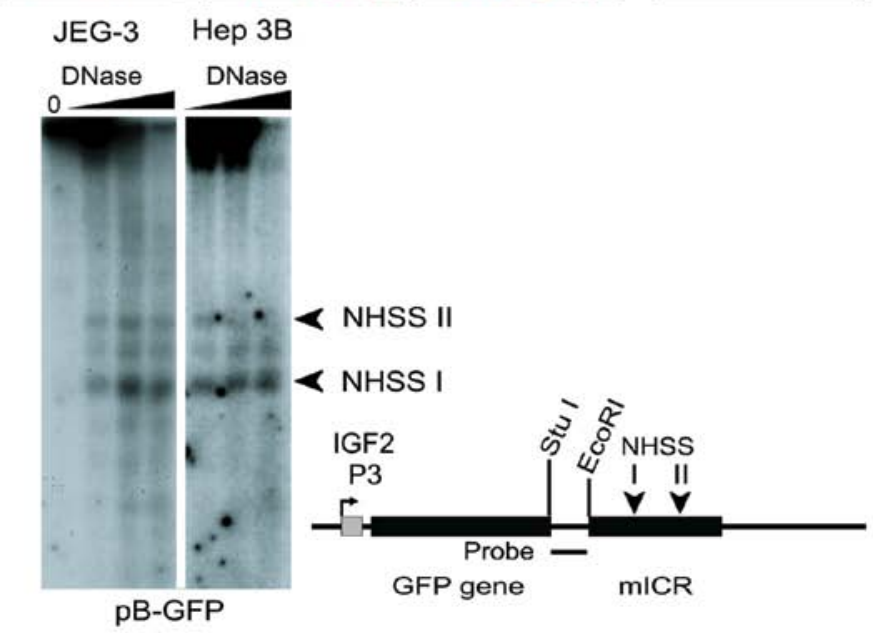

Figure 5. The insulator function of the ICR can be bypassed in human cancer cells and correlates to levels of PLAG1 expression. (A) Schematic illustrations of the GFP reporter constructs used in the insulator assays. The analysis of GFP expression was performed using flow cytometry (upper right) where the bars depicts relative GFP expression after correlation to RFP expression (pRep9RFP) and with confocal microscopy where the panels show the GFP expression pattern of the different reporter genes in a subset of cells in Hep3B and JEG-3. The left sections show GFP expression in the construct. The middle section of each panel shows the expression of the transfection control construct, DsRed2. The right sections show an overlay, where the distinctive GFP and RFP expression pattern can be determined. (B) The chromatin conformation of the transfected ICR is similar in both GFP expressing and non-expressing cells. DNase I-treated nuclei from JEG-3 and Hep3B transfected with pB-GFP, was digested with StuI. The nuclease hypersensitive sites (NHSS I and II) correspond to CTCF target sites [Kanduri et al (23)] and are identical in both cell lines.

PLAG1 induction, the levels of P3-driven GFP in the presence of insulator slightly supersede non-induced cells containing the insulator-free construct. This finding suggests that the stringency of the system normally regulated by insulator-action is partially lost in JEG-3 cells when PLAG1 is overexpressed.

\section{Discussion}

In the current study we report on a novel aspect of cell line specific IGF2 regulation involving the PLAG1 transcription factor, its binding to the $\mathrm{P} 3$ promoter region, and the possible consequence of PLAG1 expression in the context of promoter/enhancer interaction. Although, it has been shown previously by EMSA that the IGF 2 P3 promoter contains PLAG1 consensus-binding sites (19), the binding of PLAG1 to the IGF2 P3 promoter in live cells has not been reported before. We therefore investigated this by ChIP analysis. The JEG-3 and Hep3B cells were analyzed in order to verify the notion that PLAG1 is important for activity of the IGF2 P3 promoter. We show here that PLAG1 overexpression only increase binding of PLAG1 to the P3 promoter in Hep3B cells, 

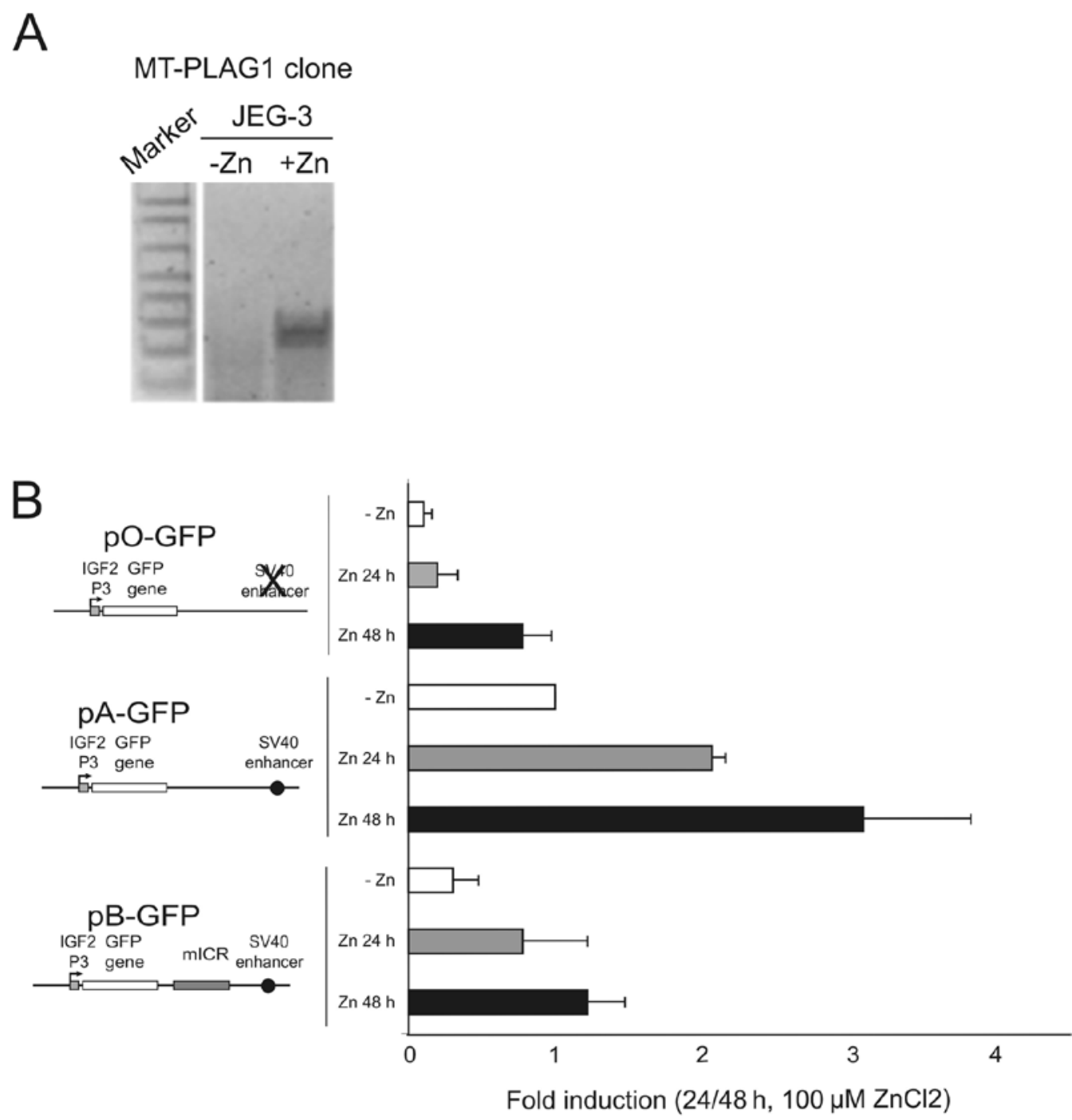

Figure 6. Expression analyses of JEG-3 cells in a stable MT-PLAG1 clone and subsequent transient IGF2-P3-GFP transfection. (A) Semi-quantitative RT-PCR analysis showing increased PLAG1 expression in the Zn-treated MT-PLAG1 JEG-3 clone (+Zn) as compared to untreated cells (-Zn). B. The MT-PLAG1 JEG-3 cell-clone was transfected with IGF2-P3-GFP reporter constructs and analysis of GFP expression was performed using flow cytometry. The bars depict relative GFP expression after correlation to RFP expression (DsRed2) and indicate fold induction of GFP expression with non-induced (-Zn) and induced (+Zn) PLAG1 expression with different reporter constructs. The expression levels of GFP was analysed after both 24 and $48 \mathrm{~h}$, showing an increased level of GFP expression after $48 \mathrm{~h}$ of induction. The expression level of the non-induced pA-GFP construct is set at 1 and is referred to as the basic state of enhanced expression. The error bars denote the SEM of three independent experiments.

leading to moderate increase of the endogenous $I G F 2$ expression in Hep3B, while neither promoter binding nor expression were affected in JEG-3 cells. On the other hand, PLAG1 stimulated P3 promoter driven GFP transcription in JEG-3 cells as well as overcame, at least in part, the H19 insulator in a GFP-plasmid reporter system.

Transcriptional insulators are specialized cis-acting elements that isolate promoters from positive and negative influences by distal enhancers. The $H 19$ upstream insulator is also an ICR, which regulates the allele-specific transcription of the IGF2 gene in a parent of origin-dependent manner. This is achieved by methylation of the ICR on the paternal allele leading to hindrance of the insulator protein CTCF and loss of communication between the shared $H 19 / I G F 2$ enhancer downstream of $H 19$ and the IGF2 promoter(s) (12). Methylation of the ICR also spread into the $H 19$ promoter leading to transcriptional silencing. Conversely, the unmethylated ICR on the maternal allele binds CTCF and then functions as an enhancer-blocking insulator that prevents expression of the maternal IGF2 allele.

Our findings suggest a new role for PLAG1 in IGF2 overexpression in cancer. Not only does PLAG1 expression affect the activity of the promoter, it also appears to increase the effect of enhancer activated transcription. The function of PLAG1 as a regular transcription factor and proto-oncogene has been reported previously, and there is previous evidence to show that it binds to a consensus binding site in the IGF2 P3 promoter and activates its transcription in vitro. We propose, however, that PLAG1 has additional, more complex functions, one of which may be to act as a promoter/enhancer facilitator. The reporter constructs used in this study were designed to interrogate the influence of insulator function of the H19 ICR on IGF2 P3 promoter activity in cells with different levels of PLAG1 expression. We observed that JEG-3 cells are relying on an exogenous PLAG1 expression to overcome the silencing 
effect of the insulator in the construct, although they do transcribe low levels of PLAG1 mRNA. Hep3B cells on the other hand, which have a considerably higher endogenous PLAG1 expression, are already insensitive to the insulator. In this connection, it is important to note that the chromatin structure of the ICR in the episomal construct, was found to be similar in both JEG-3 and Hep3B cells (Fig. 5B), indicating presence of binding by CTCF, and thus promoting insulation, in both cases. In this scenario the induction of PLAG1 was apparently able to partially overcome an active insulation since the ICR containing construct ( $\mathrm{pB}-\mathrm{GFP}$ ) showed a substantially higher GFP expression than the enhancer-free and ICR-free construct (pO-GFP), after PLAG1 induction (Fig. 6B). The inability to completely overcome insulation was evident, however, since PLAG1 expression in the ICR containing construct did not reach the GFP expression level of the ICR-less/enhancer containing construct (pA-GFP).

It is unknown why the low endogenous PLAGl expression in JEG-3 cells does not affect the expression of the GFP reporter. It is possible that a threshold concentration of PLAG1 is required to push a transcription factor complex towards assembly.

Considering that in the GFP reporter construct, PLAG1 is able to influence the IGF2 P3 promoter activity in JEG-3 cells, it is challenging to understand why the functional response to PLAG1 is different from that in the endogenous P3 promoter. Maybe PLAG1 binding in the endogenous IGF2 P3 promoter requires additional factors, absent in JEG-3 cells but present in Hep3B. Although we did demonstrate that highly overexpressed PLAG1 actually increased binding to endogenous P3 in Hep3B cells, only moderate activation of IGF2 transcription was achieved and non-transfected cells did not demonstrate substantial endogenous PLAG1 binding to the P3 promoter suggesting that other factors, not excluding chromatin structure, are important for the endogenous IGF2 transcription. This was in contrast to the JEG-3 cells where neither binding nor activation could be demonstrated despite substantial PLAG1 expression. These cells are apparently not geared at all for PLAG1 directed IGF2 expression, and since the GFP-reporter construct was PLAG1 inducible, chromatin structure in the endogenous gene may be an important factor. The lack of additional IGF2 activation in Hep3B cells after PLAG1 overexpression may also be due to an already saturated promoter by other permissive transcription factors, or lack of required additional factors. The PLAG1 protein may also be sumoylated and thus inactivated, as was shown by others (25). To our knowledge, whether or not sumoylation of PLAG1 influences its binding to the IGF2 $\mathrm{P} 3$ promoter has not been demonstrated. It is also possible that overexpression of PLAG1 does not stimulate the promoter P3-dependent transcription of IGF2 in JEG-3 cells, due to methylation mediated inactivation of the P3 promoter or the H19 DMR. These questions are currently being analyzed.

Although it would be a possibility that PLAG1 interferes with allele-specific expression of $I G F 2$ by interfering with the ICR, we did not study this issue here. In addition, Declercq et al concluded that only the paternal Igf2 allele is involved in the PLAG1 induced formation of pleomorphic adenomas of the salivary glands, in a transgenic mouse model (26). This suggests that although PLAG1 in certain contexts may interefere with the H19 ICR, it is not involved in the imprinting mechanism.
In conclusion, we show here that the role of PLAG1 in activation of $I G F 2$ is context-specific in that it readily activates an episomal reporter driven by the IGF2 $\mathrm{P} 3$ promoter, it is cell type-specific in its sensitivity to promoter enhancer insulation and in the endogenous context, and that both binding to the IGF2 $\mathrm{P} 3$ promoter and endogenous transcription activation is cell type-specific. We also suggest that PLAG1 may act as a facilitator since it partially overcomes the insulator function of the H19 DMR, at least in vitro.

\section{Acknowledgements}

This study was financially supported by the Swedish Cancer Society, the Swedish Research Council, the Swedish Society for Medical Research, the Stockholm County Council, and funds from Karolinska Institutet. We would like to greatly acknowledge Marianne Voz for the Zn-inducible PLAG1 plasmid construct, and Katharina Wimmer for the PLAG1 expression vector.

\section{References}

1. LeRoith D and Roberts CT Jr: The insulin-like growth factor system and cancer. Cancer Lett 195: 127-137, 2003.

2. Cui H: Loss of imprinting of IGF2 as an epigenetic marker for the risk of human cancer. Dis Markers 23: 105-112, 2007.

3. Cui H, Cruz-Correa M, Giardiello FM, et al: Loss of IGF2 imprinting: a potential marker of colorectal cancer risk. Science 299: 1753-1755, 2003.

4. Feinberg AP, Cui $\mathrm{H}$ and Ohlsson R: DNA methylation and genomic imprinting: insights from cancer into epigenetic mechanisms. Semin Cancer Biol 12: 389-398, 2002.

5. Ohlsson R, Kanduri C, Whitehead J, Pfeifer S, Lobanenkov V and Feinberg AP: Epigenetic variability and the evolution of human cancer. Adv Cancer Res 88: 145-168, 2003.

6. Steenman MJ, Rainier S, Dobry CJ, Grundy P, Horon IL and Feinberg AP: Loss of imprinting of IGF2 is linked to reduced expression and abnormal methylation of $\mathrm{H} 19$ in Wilms' tumour. Nat Genet 7: 433-439, 1994.

7. Cui H, Horon IL, Ohlsson R, Hamilton SR and Feinberg AP: Loss of imprinting in normal tissue of colorectal cancer patients with microsatellite instability. Nat Med 4: 1276-1280, 1998.

8. Kaffer CR, Srivastava M, Park KY, et al: A transcriptional insulator at the imprinted H19/Igf2 locus. Genes Dev 14: 1908-1919, 2000.

9. Holmgren C, Kanduri C, Dell G, et al: CpG methylation regulates the Igf2/H19 insulator. Curr Biol 11: 1128-1130, 2001.

10. Kurukuti S, Tiwari VK, Tavoosidana G, et al: CTCF binding at the H19 imprinting control region mediates maternally inherited higher-order chromatin conformation to restrict enhancer access to Igf2. Proc Natl Acad Sci USA 103: 10684-10689, 2006.

11. Pant V, Mariano P, Kanduri C, et al: The nucleotides responsible for the direct physical contact between the chromatin insulator protein CTCF and the H19 imprinting control region manifest parent of origin-specific long-distance insulation and methylation-free domains. Genes Dev 17: 586-590, 2003.

12. Yoon YS, Jeong S, Rong Q, Park KY, Chung JH and Pfeifer K: Analysis of the H19ICR insulator. Mol Cell Biol 27: 3499-3510, 2007.

13. Nativio R, Wendt KS, Ito Y, et al: Cohesin is required for higherorder chromatin conformation at the imprinted IGF2-H19 locus. PLoS Genet 5: e1000739, 2009.

14. Cui H, Niemitz EL, Ravenel JD, et al: Loss of imprinting of insulin-like growth factor-II in Wilms' tumor commonly involves altered methylation but not mutations of CTCF or its binding site. Cancer Res 61: 4947-4950, 2001.

15. Ulaner GA, Vu TH, Li T, et al: Loss of imprinting of IGF2 and H19 in osteosarcoma is accompanied by reciprocal methylation changes of a CTCF-binding site. Hum Mol Genet 12: 535-549, 2003 . 
16. Cui $\mathrm{H}$, Onyango $\mathrm{P}$, Brandenburg S, Wu Y, Hsieh CL and Feinberg AP: Loss of imprinting in colorectal cancer linked to hypomethylation of H19 and IGF2. Cancer Res 62: 6442-6446, 2002.

17. Takai D, Gonzales FA, Tsai YC, Thayer MJ and Jones PA: Large scale mapping of methylcytosines in CTCF-binding sites in the human H19 promoter and aberrant hypomethylation in human bladder cancer. Hum Mol Genet 10: 2619-2626, 2001.

18. Kas K, Voz ML, Roijer E, et al: Promoter swapping between the genes for a novel zinc finger protein and beta-catenin in pleiomorphic adenomas with $\mathrm{t}(3 ; 8)(\mathrm{p} 21 ; \mathrm{q} 12)$ translocations. Nat Genet 15: 170-174, 1997.

19. Voz ML, Agten NS, Van de Ven WJ and Kas K: PLAG1, the main translocation target in pleomorphic adenoma of the salivary glands, is a positive regulator of IGF-II. Cancer Res 60: 106-113, 2000.

20. Zatkova A, Rouillard JM, Hartmann W, et al: Amplification and overexpression of the IGF2 regulator PLAG1 in hepatoblastoma. Genes Chromosomes Cancer 39: 126-137, 2004.

21. Astrom A, D'Amore ES, Sainati L, et al: Evidence of involvement of the PLAG1 gene in lipoblastomas. Int J Oncol 16 1107-1110, 2000.
22. Landrette SF, Kuo YH, Hensen K, et al: Plag1 and Plagl2 are oncogenes that induce acute myeloid leukemia in cooperation with Cbfb-MYH11. Blood 105: 2900-2907, 2005.

23. Kanduri C, Holmgren C, Pilartz M, et al: The 5' flank of mouse H19 in an unusual chromatin conformation unidirectionally blocks enhancer-promoter communication. Curr Biol 10: 449-457, 2000.

24. Dahl JA and Collas P: Q2ChIP, a quick and quantitative chromatin immunoprecipitation assay, unravels epigenetic dynamics of developmentally regulated genes in human carcinoma cells. Stem Cells 25: 1037-1046, 2007.

25. Van Dyck F, Delvaux EL, van de Ven WJ and Chavez MV: Repression of the transactivating capacity of the oncoprotein PLAG1 by SUMOylation. J Biol Chem 279: 36121-36131, 2004.

26. Declercq J, van Dyck F, van Damme B and van de Ven WJ: Upregulation of Igf and Wnt signalling associated genes in pleomorphic adenomas of the salivary glands in PLAG1 transgenic mice. Int J Oncol 32: 1041-1047, 2008. 